\title{
A New Insight for Faraday's Law
}

\author{
Narahari V. Joshi \\ Department of Physics, University of Los Andes, Merida, Venezuela \\ Email: joshi@ula.ve
}

Received 28 September 2014; revised 24 October 2014; accepted 15 November 2014

Copyright (C) 2014 by author and Scientific Research Publishing Inc.

This work is licensed under the Creative Commons Attribution International License (CC BY).

http://creativecommons.org/licenses/by/4.0/

c) (i) Open Access

\section{Abstract}

The origin for one of the fundamental laws of electromagnetism, namely Faraday's law is explained for the first time on the basis of the presence of strings in the form of a compact liquid. The rate of change of the magnetic field produces a pressure gradient in the medium giving rise to the fluid flow. According to fluid dynamics, the stress and the gradient of strain are originated in the space which creates vibrations in the system and is related with the electric field. The details of the mechanism which produces a circular motion of the electric field, $\nabla X E$, are also addressed.

\section{Keywords}

Faraday's Law, Fluid Dynamics, Magnetic Field

\section{Introduction}

Faraday's law of induction is one of the basic principles of electromagnetism which shows that the change in the magnetic field produces an electric field. The electric field in the space is originated when there is a change in the strength of the magnetic field. Transformers, inductors, and many types of electrical motors and generators are based on this principle [1]. The observed phenomenon and its details are explained with the help of Maxwell's equation given by [2]

$$
\frac{\mathrm{d} B}{\mathrm{~d} t}=-\nabla X E
$$

In spite of its importance in the field of electromagnetism and its applications in modern technology, the origin for this phenomenon is not known. It has never been discussed how and why the variation in the magnetic field gives rise to the electric field in the form of rotational energy. This most significant principle needs to be examined in order to understand the mechanism behind it. The purpose of the present investigation, therefore, is to provide a foundation for this well established principle.

The close relationship between electric and magnetic fields and several aspects of Maxwell's equation have 
been discussed earlier by Joshi [3] on the basis of the presence of vibrating strings in the space. In this context, strings are considered as a compact, incompressible, nonviscous, surface tension free liquid. It is revealed that several fundamental aspects of electromagnetism turn out to be natural consequences of fluid dynamics. For examples, the mechanisms for the force of electrostatic repulsion (or attraction) [4] and the foundation for Lorentz force have also been explained satisfactorily with this novel approach [5].

An important aspect of the present theory is that the change in the density of the magnetic field in the space gives rise to the electric field. In fact, the change in the magnetic field, $\frac{\mathrm{d} B}{\mathrm{~d} t}$, in an incompressible fluid creates several significant alterations in the fluid.

\section{Fluid Dynamics}

The magnetic field is associated with the lines of forces and its intensity corresponds to the density of the magnetic field. The change in the density creates a variation in the pressure in the space which is filled with strings. It is, therefore, necessary to examine the variation of pressure in the fluid [6].

Let $P_{e}$ be the pressure at equilibrium and $P_{B}$ be the pressure caused at any point due to the variation in the magnetic field. The pressure in the fluid has two parts. One is isotropic and the other a non-isotropic part which is known as deviatoric stress tensor and depends mainly on the spatial distribution of the velocity. This tensor gives rise to spatial properties originated from the motion of the fluid. The variation in the magnetic field originates the deviatoric pressure. In the present context we will address mainly to it. The pressure gradient $\nabla P[6]$, is given by

$$
\nabla P(x, y, z)=P_{B}-P_{e}
$$

The variation of pressure is not localized at any particular point; instead, it is spread in three dimensional space. The pressure difference associated with the changes in the magnetic field varies from place to place, both in magnitude and in direction. The energy density, therefore, is altered dramatically and instantaneously, originnating a motion in the fluid. It is assumed that $\nabla P(x, y, z)$, depends only upon the instantaneous local velocity gradient and it is a linear function of various tensor components .

In the present case, the incompressible liquid has considerable resistance for the translation motion and therefore, the pressure difference gives rise to strain in a specific part of the liquid. As mentioned earlier, the magnetic field (or lines of forces) is an organized collective rotational motion of strings [3], the pressure difference causes stress and the velocity of the fluid depends upon its position [6].

$$
\mathrm{d} V_{i}\left(x_{j}\right)=\frac{\partial V_{i}}{\partial X_{j}} \partial X_{j}
$$

However, the velocity $V(x, y, z)$ is directly proportional to the strength of the magnetic field $B$ and therefore $V=k \nabla P\left(x_{j}\right)$. Here $\mathrm{k}$ is a constant of proportionality.

As both the velocity and the displacement $\partial X_{j}$ are vectors, $\frac{\partial V_{i}}{\partial X_{j}}$ is a second order tensor and it can be separated into symmetric and antisymmetric components [7]

$$
\mathrm{d} V_{i}=\frac{1}{2}\left[\frac{\partial V_{i}}{\partial X_{j}}+\frac{\partial V_{j}}{\partial X_{i}}\right] \partial X_{j}+\frac{1}{2}\left[\frac{\partial V_{i}}{\partial X_{j}}-\frac{\partial V_{j}}{\partial X_{i}}\right] \partial X_{j}
$$

As the magnetic field varies, both parts of Equation (4) become time dependent and they play a significant role in the present theory. First, let us examine the ant symmetric part of Equation (4) which plays an important role in the rotational process. It is given by a tensor $\xi_{i j}$,

$$
\xi_{i j}=\frac{1}{2}\left[\frac{\partial V_{i}}{\partial X_{j}}-\frac{\partial V_{j}}{\partial X_{i}}\right]
$$

These are the components of $(\nabla X V)$ suggesting a rotational motion in a compact liquid of strings. The 
above analysis shows that the variation in the magnetic lines of forces produces a rotational motion in the liquid.

The other important aspect of the tensor analysis is the strain in the system created by the first part of the tensor which is symmetric. The magnitude of the stress tensor varies in the space from point to point according to the intensity of the magnetic field (or density of lines of force) and therefore, a strain gradient is created which has important consequences in the fluid dynamics.

The stress tensor, in a strong non equilibrium state of incompressible, high density liquid needs to be examined carefully.

\section{Tensors in Fluid}

The stress tensor in a liquid at the equilibrium state is given by [6]

$$
\sigma_{i j}=-P_{e} \delta_{i j}
$$

The negative sign indicates that the pressure is quizzing. Here $P_{e}$ is the static equilibrium pressure in the liquid and it is a function of the position. However, in the present case Equation (6) is invalid because of the presence of the variation in the magnetic field and the motion of the fluid, particularly in the circular form. Shear and non uniform tangential stress is non zero and the normal component of stress depends upon the direction and, unlike liquid at rest, the pressure is not acting equally in all directions.

The total stress at any time; therefore, becomes

$$
\sigma_{i j}=-P_{e} \delta_{i j}+d_{i j}
$$

where $d_{i j}$ is the deviatoric stress tensor and it is non isotropic (depends upon the direction). It is originated mainly from the flow of liquid which creates the stress gradient [6].

In non equilibrium states; the present kinetic theory models do not predict or carry out a precise calculation. In this case, it is not possible to estimated an accurate formalism for the deviatoric stress tensor, $d_{i j}$. It is assumed that $d_{i j}$ mainly depends upon the velocity gradient and then

$$
d_{i j}=\xi_{i j k l} \frac{\partial V_{k}}{\partial X_{l}}
$$

Here $\xi_{i j k l}$ is the tensor of the fourth rank. The Equation (8) helps to understand the role of the velocity gradient with the deviatoric stress tensor.

The velocity gradient contributes in two different ways. First it produces a contraction (or expansion) in the liquid and second it gives rise to shears stress. In the present system the first possibility does not exist. For strings in the form of a compact and incompressible liquid, a reduction in size is very difficult to imagine. Therefore, the only possibility is the formation of shear stress.

The strain originated in the fluid is strongly related with the gradient of the velocity $\frac{\partial V_{k}}{\partial X_{l}}$. The relation is complex as the fluid is in motion. In this case, the flow of energy and transport of the momentum also contributes, up to a certain extent, in the variation of stress.

In the present case, we are dealing with a very special and uncommon type of fluid-a compact form of strings. It is isotropic when we consider the character of every element, however, because of the magnetic field; there exists an organized rotational motion of the fluid. The energy corresponding to the magnetic lines of forces creates a "space structure" which is not observed in normal fluids. This does not affect the flow field; however, strains in the liquid will have different specification. In this sense the fluid is not isotropic. The strain originated from the deviatoric tensor, $d_{i j}$, might not be symmetrical also. Some of the off diagonal elements could be negligibly small. This depends upon several factors among them are the direction and intensity of the variation of the magnetic field.

At any point in space, the magnetic field varies in time, both in magnitude and in direction. Obviously, it creates a very complicated situation as far as the tensile strain is considered. It varies from place to place. For simplicity, it can be assumed that the variation due to the transport of the momentum is not so significant as compared with the strain originated from the gradient of the velocity. Let $S_{k}$ be the displacement per unit of length in the $k$ direction corresponding to the strain. Then, $\frac{\partial S_{k}}{\partial X_{l}}$ is proportional to $\frac{\partial V_{k}}{\partial X_{l}}$. The constant of pro- 
portionality depends upon the parameter of the elements of the fluid and the organized rotational motion of the strings which are affected.

In this case Equation (8) becomes

$$
d_{i j}=-C_{i j k l} \frac{\partial S_{k}}{\partial X_{l}}
$$

$C_{i j k l}$ is the proportionality tensor. Equation (9) is Hook's law of elasticity [7] which indicates the creation of energy associated with harmonic oscillators.

\section{Discussion}

The role of the gradient of stress in vibrational analysis has been studied extensively in solids of different dimensions of micro plates (rectangular and circular forms). It is found that, apart from the properties of the material constants, the vibrational frequency depends upon the size of the sample. Smaller the sample, higher is the natural frequency [8] [9]. Thus, it is natural to expect that strings of much smaller dimensions $\left(10^{-33} \mathrm{cms}\right)$ can have a higher frequency induced by the variation in the magnetic field. Certainly, more investigation in this direction will be a great help.

Such investigation has been extended to the micro tube through which the liquid is flowing [8]. The gradient of stress produces vibrations in the liquid and hence in the micro tubes [10]. The frequencies depend upon the inner dimensions, other mechanical properties of the tubes and also on the velocity of the flow of the liquid. An important aspect is that the frequency is found to be very sensitive to the velocity of the fluid suggesting that it generates vibrations in the nearby region and they are detected even in fluid conveying micro-tubes [8] [9]. Moreover, experimentally, it is found that smaller the flow velocity, higher is the excited frequency [8]. These experimental details confirm that the flow of the liquid generates the vibrations in the system; slower the translational motion, higher is the frequency.

Harmonic oscillations can also be generated in the system when the strain is function of time or the medium has a possibility to store the energy and acts as a buffer. In the present situation, both requirements are fulfilled and Equation (9) indicates that the additional harmonic oscillations are generated in the liquid composed of vibrating strings due to the variation in the magnetic field. According to quantum field theory, harmonic oscillations and fields are strongly correlated. The vibrations are not localized but spread all over the space, the intensity varies from point to point according to the density of the lines of forces.

Dynamics of energy transfer and its relation with vibrational and rotational energy of the elements of the liquid has been studied extensively both theoretical and experimentally. Infrared and Raman spectroscopy have been employed for this purpose and it is found that vibrational and translational-rotational modes become active [11]. Various interpretations are given to explain the appearance of extra vibrational modes in liquids and they are associated with pseudo-lattice oscillations or intra molecular vibrations. This effect is strong, particularly, in a dense and compact liquid [12].

The above discussion clearly indicates that the magnetic lines of force of variable density create stress and consequently strain in the compact liquid formed by strings. Recent investigation also revealed that the strain gradient excites torsion-free vibrational modes in elements of the fluid [13]. An extensive study in this direction is required to examine the parameters involved and the limitations on the boundary conditions. In the present case, the magnetic field rotates the mass of the fluid (as shown in Equation 4) and simultaneously generates vibrations in the neighboring region.

Now, let us examine the rotational flow of the fluid given by $(\nabla X V)$. The velocity of the fluid is proportional to the magnetic field $B\left(\frac{\text { mass }}{\text { Sec. }} \cdot \frac{1}{\text { Coulomb }}\right)$ and the rate of it’s variation. The magnitude of the strain or "deformation length" caused in a magnetic field and the vibrational frequency (Cycles/sec), excited by the gradient of strain is directly proportional to the rate of change of magnetic field. Therefore, the energy flow associated with the flow field originated by $\frac{\mathrm{d} B}{\mathrm{~d} t}$ can be expressed as

$$
\text { [ } \frac{\text { mass. }}{\text { seg }} \frac{1}{\text { Coulomb }} \frac{\text { cycles }}{\text { seg }} \text { “Deformation length”] }
$$


Hence,

$$
\frac{\mathrm{d} B}{\mathrm{~d} t}=-\nabla X\left(\frac{\text { mass. }}{\mathrm{seg}} \frac{1}{\text { Coulomb }} \frac{\text { cycles }}{\mathrm{seg}}\right. \text { "deformation length"). }
$$

Here the "deformation length" is not refereed to the alteration in the constituent elements of the fluid (strings); but it is referred to the modification in the organized structure of the fluid. According to the intensity of vibrations and the symmetry of the system, the structure is altered and reorganized. "The deformation of the length" is directly proportional to the velocity with which the magnet (or magnetic field) is moving and hence it might be proportional to the intensity or amplitude of the field. The quantity shown in the bracket of Equation (10) has exactly the dimensions of the electric field E. The most important aspect is that the energy associated with the electric field is related to the vibrations originated from the variation in the magnetic field. This confirms an earlier exploration where the electric field is associated with the collective excitation of the vibrational energy [3]; meanwhile, the magnetic field is related with collective rotational motion.

The above mentioned relation between stress-strain and electric field is not a new concept. Piezoelectric effect is based on these parameters indicating that the generation of pressure gradient gives rise to the electric field due to the reorientation or reconfiguration of the system (for a crystal with lacking symmetry. some liquids, ceramic etc.). The close relation of strain with piezoelectricity is extensively discussed [14] and the relation between strain and electric field is confirmed.

In the present case, the strain is not in a system of solid or ceramics but it is in the compact fluid of strings where "pseudo structure" is altered with the pressure. Equation (10), therefore, turns out to be Faradays Law given by Equation (1).

The electric field (lines of force) is originated from the presence of the charge particle, (e.g. electron or proton) and it is some what difficult to understand why the variation of the magnetic field creates an electric field as if the charge particle is present nearby. This dilemma is already explained [3] by considering that the electron is formed by strings with a specific vibrational frequency and it excites the vibrations in an organized manner, following the laws of symmetry, in a space filled with strings. In the present case, the magnetic field is altered in the space and creates strain and hence the vibrations are excited.

However, a detailed analysis and estimation of the magnitude of the parameters are not possible to estimate because they are directly related with the density and other properties of the vibrating strings, their collective rotational and vibrational motions. The energy of vibrating strings plays a crucial role in the electric field and how much the deformation strain contributes in the vibrational frequency is difficult to estimate at this stage. In addition to this, the vibrating energy of strings acts as a storage or buffer for the energy conversion process. A significant aspect is that when the magnetic field is multiplied by the frequency of vibration (cycles/sec) together with the "deformation length" it turns into electric field. Further insight in this direction might throw light on the fundamental properties of strings and their interactions in the context of electric and magnetic fields.

The present approach is consistent with quantum field theory according to which fields are associated with vibrations and particles are localized vibrations of the corresponding field [15]. A physical electron is a localized vibration which induces vibrations in the surrounding strings according to the basic principles of symmetry. In the present case, vibrations are originated from the strain created by the variation of the magnetic field (or pressure in the liquid of compact strings). Even though the result is the same, the mechanism behind the development of the electric field is different. This permits to extend our views to the subatomic world where different particles (like electron, muon, gluon) have corresponding fields and interact in specific ways.

\section{Conclusion}

In the present investigation, we want to show how the variation in the magnetic field produces $(\nabla X E)$ and how the gradient of strain tensor plays a crucial role. The most important aspect is that the vibrational frequency is generated from the variation in the magnetic field.

\section{References}

[1] Ulbay, F.T. (2007) Fundamentals of Applied Electromagnetic. Prentice Hall, New Jersey.

[2] Feynman R.P., Leighton R.B. and Sands, M. (1969) The Feynman Lectures on Physics. Vol. 2, Addision Wesley, Boston. 
[3] Joshi, N.V. (2013) Energy Conversion from the Vacuum Field to Electromagnetic Fields. Physics Essays, 26, 61-67.

[4] Joshi, N.V. (2013) Mechanism for Electrostatic Repulsion or Attraction. World Journal of Mechanics, 3, 307-309.

[5] Joshi, N.V. (2014) A Foundation for Lorentz Force Based on Vibrating Strings as a Compact Fluid. Word Journal of Mechanics, 4, 247-250.

[6] Batchelor, C.K. (1993) An Introduction to Fluid Dynamics. Cambridge Press, Cambridge.

[7] Arfken, G. (1985) Mathematical Methods for Physicist, Academic Press, New York.

[8] Wang, L. (2010) Size Dependent Vibration Characteristic of Fluid Conveying Micro-Tubes. Journal of Fluid Dynamics, 26, 675-684.

[9] Jomehzadeh, E., Noori, H.R., Saidi, A.E. and Physica, E. (2011) The Size Dependent Vibration Analysis of MicroPlates Based on a Modified Couple Stress Theory, 43, 877-883.

[10] Yang, M. (2012) Dynamics of Resonant Energy Transfer in OH Vibrations of Liquid Water. Bulletin of the Korean Chemical Society, 33, 885-892. http://dx.doi.org/10.5012/bkcs.2012.33.3.885

[11] Warrier, A.V.R. and Krimm, S. (1970) Intermolecular Vibrations in Liquid Secondary Chlorides. The Journal of Chemical Physics, 52, 4316-4317. http://dx.doi.org/10.1063/1.1673649

[12] Wang, L. (2011) Vibration Analysis of Nanotubes Conveying Fluid Base Gradient Elasticity Theory. Journal of Vibration and Control, 18, 313-320. http://dx.doi.org/10.1177/1077546311403957

[13] Narendhar, S., Ravinder, S. and Gopalakrishnan, S. (2012) Strain Gradient Torsional Vibration Analysis of Micro and Nano Rods. International Journal of Nano Dimension, 3, 1-17.

[14] Gautschi, G. (2002) Piezoelectric Sensoric. Springer, Berlin. http://dx.doi.org/10.1007/978-3-662-04732-3

[15] McMahon, D. (2008) Quantum Field Theory. McGraw-Hill, New York. 
Scientific Research Publishing (SCIRP) is one of the largest Open Access journal publishers. It is currently publishing more than 200 open access, online, peer-reviewed journals covering a wide range of academic disciplines. SCIRP serves the worldwide academic communities and contributes to the progress and application of science with its publication.

Other selected journals from SCIRP are listed as below. Submit your manuscript to us via either submit@scirp.org or Online Submission Portal.
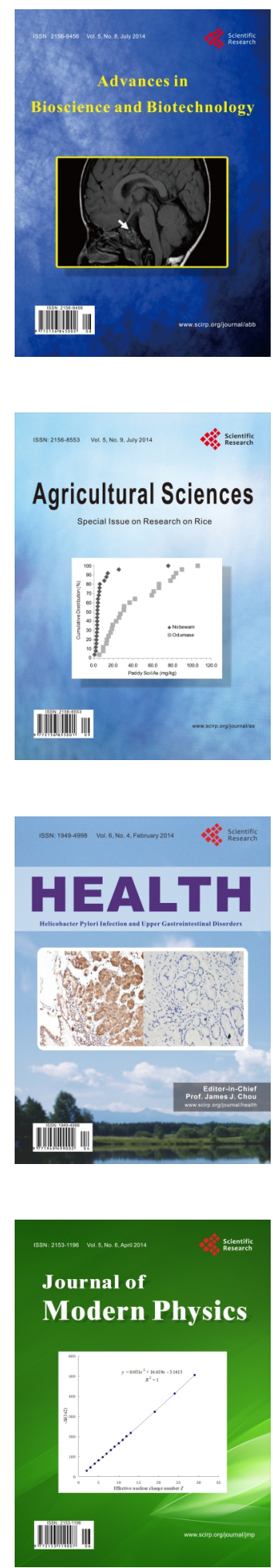
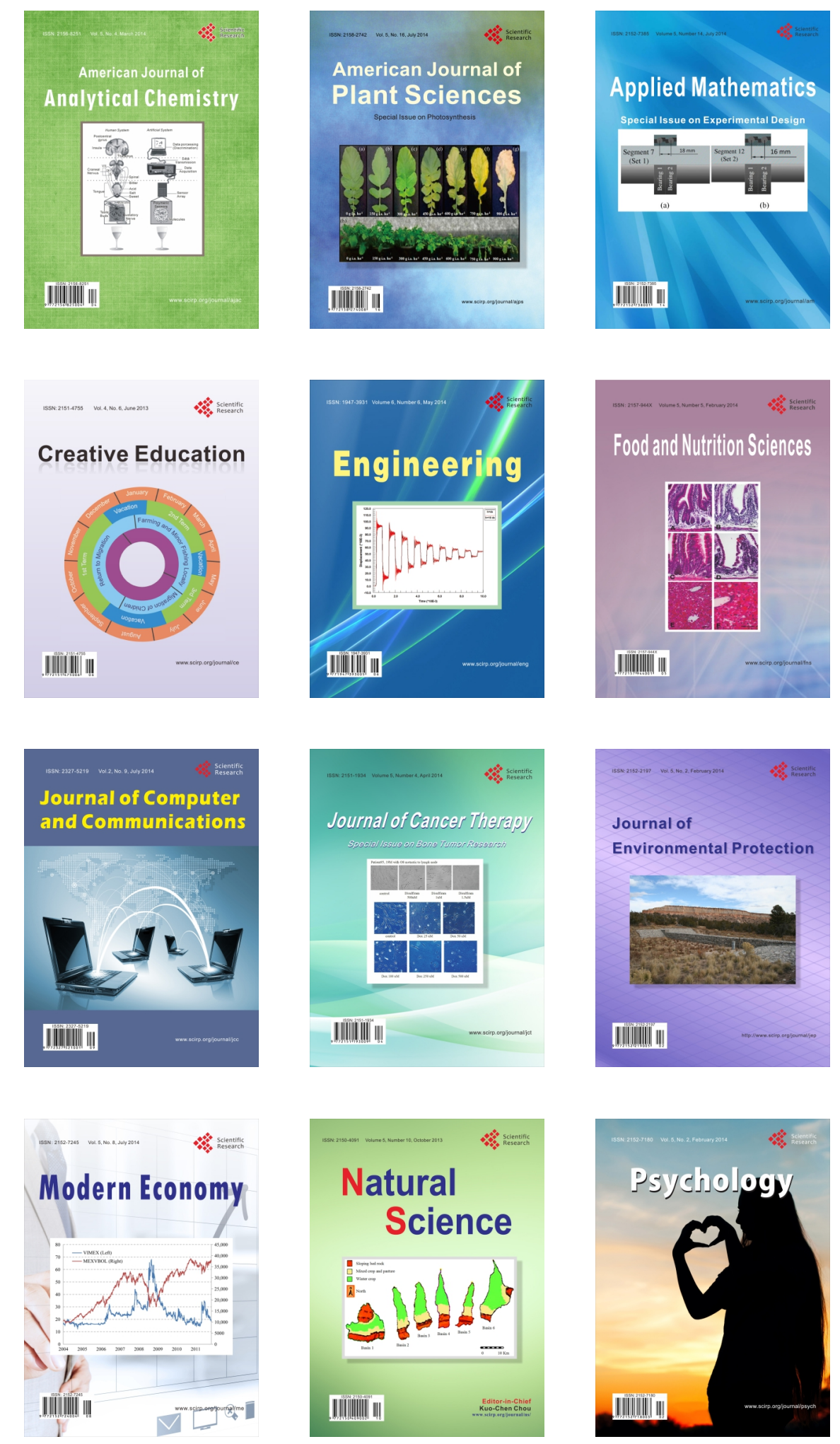